\title{
Modelling of localised gas preferential pathways in claystone
}

\author{
Pierre Gerard ${ }^{1}$, Jon Harrington ${ }^{2}$, Robert Charlier $^{3}$, Frédéric Collin ${ }^{3}$
}

\author{
${ }^{1}$ Université libre de Bruxelles (ULB), BATir Department (Building, Architecture and Town \\ planning), Laboratory of Geomechanics, Brussels, Belgium (piergera@ulb.ac.be) \\ Av. F. Roosevelt, 50 - CPI 194/02 \\ 1050 Bruxelles \\ Belgium \\ ${ }^{2}$ British Geological Survey, Keyworth, UK (jfha@bgs.ac.uk) \\ ${ }^{3}$ Université de Liège (ULg), ArGEnCo Department, Liege, Belgium \\ (Robert.charlier@ulg.ac.be;f.collin@ulg.ac.be)
}

\begin{abstract}
A long-term injection gas test on initially saturated claystone samples under isotropic confining pressure is modelled in a $2 \mathrm{D}$ hydro-mechanical framework, which includes the hydraulic anisotropy. Evidences of localised pathways through the sample have been observed experimentally, which are difficult to reconcile with standard 2 phase flow models. The presence of an embedded pre-existing fracture is included in a continuum finite element model. A hydro-mechanical coupling between the fracture aperture, permeability and the retention properties along the fracture is included in the model. Thanks to the increase in permeability and the decrease of the air entry pressure induced by the rise in fluid pressure at constant mean total stress, the model provides a good agreement with the experimental observations. The discussion offers additional insight into the fluid flow mechanisms into the sample and the processes involved in the development of localised gas pathways. This study allows conclusions to be drawn regarding the performance of the model and its practical limitations.
\end{abstract}

Keywords: gas migration, claystone, unsaturated rock, numerical modelling 


\section{Introduction}

Argillaceous rocks are generally considered as suitable geological barriers in radioactive waste disposal as well as in $\mathrm{CO}_{2}$ storage in deep formations. Their low permeabilities and retention capabilities offer interesting properties to limit the migration of the radionuclides released from the waste. In the field of radioactive waste confinement, the question of gas transfer in argillaceous formations is a crucial issue. Following repository closure, significant quantities of gases may be produced by the deterioration of the disposal components. It is important to understand the impact of gas production and migration on repository components, especially the clay barriers, in order to be able to predict the system behaviour with confidence over long time periods [1].

As suggested by Marshall et al. [2], different phenomenological processes can be defined to describe gas flows in low-permeability materials. When the gas production rates remain limited, diffusive transport of gas dissolved in pore-water may be adequate to prevent formation of a gas phase. However, if the rate of gas production exceeds the solubility limit of the interstitial fluid, a gaseous phase will be created. Under these conditions, gas will accumulate and visco-capillary flow may take place in the porous medium.

As gas pressures increase, complex behaviour for gas flow is generally observed. In laboratory studies performed on clay-based materials, gas flow is often associated with instabilities suggesting a dynamic network of conductive pathways. A common observation is that gas begins to flow once it has reached a given pressure (the breakthrough pressure), either when gas flow rate is controlled [3-7] or when gas pressure is imposed [3;8-10]. This behaviour is often interpreted as an indication of the opening of localised gas pathways inside the matrix [2].

Field investigations have been also performed to investigate the gas migration on clay host rock in various underground research laboratories [2;11-13]. While these observations can be difficult to analyse and compare with laboratory observations (because of the scale of the processes and uncertainties associated with characterisation of the boundary conditions), gas breakthrough phenomena have been observed $[2 ; 11]$. These experiments have suggested that the test results are very sensitive to minor features, for example the presence of bedding joints or interfaces between materials.

Classic concepts of porous medium two-phase flow are inappropriate to model such gas flows [14-15]. Some contributions propose modelling tools to take into account the development of localised gas paths through the claystone. The lattice models views the porous 
medium as a series of capillary networks superimposed on each other with different radius and density [16-18]. Discrete fracture networks also provide a solution to represent the effective conductivity of fractured rock at a grid-block scale [19]. Porous medium models which include some form of heterogeneity associated with a number of intrinsic characteristics (permeability, porosity) also seems to provide a solution to explain such behaviour [20].

An alternative proposition dealing with equivalent continuum medium is to associate the development of advective gas pathways with gas migration through localised fractures predefined within the material. The effect of the presence of these discontinuities can be taken into account by coupling the aperture of the discontinuity and its equivalent permeability. The opening of the fracture can be induced by hydro-mechanical loadings (e.g. rise in fluid pressure, effective unloading of the rock). This hydro-mechanical coupling, referred to as an embedded fracture model, has been proposed by Olivella and Alonso [21] to explain gas flow mechanisms in plastic clays. Initially this model has been successfully applied in the modelling of laboratory gas injection tests under shear [21]. The embedded fracture model provides also a good representation of the gas permeability evolution along interfaces between two materials or along bedding joints as a response to stress and strain changes, at both the field scale $[21 ; 22]$ and the laboratory scale [6;22]. Collectively this work suggests that it is often necessary to consider small-scale spatially heterogeneities within the material when describing the initiation of gas flow.

To contribute to a better understanding of the processes associated with the development of localised gas pathways in claystone, the modelling of a long-term laboratory gas injection test using an embedded fracture model is studied. This experiment was performed by the British Geological Survey on Callovo-Oxfordian claystone (COx), the proposed host rock for the French radioactive waste repository [23;24]. The purpose of this test was to investigate the mechanisms governing gas migration in the COx. Results from this study indicate the movement of gas is through a localized network of pathways within the claystone whose properties vary temporarily and spatially within the claystone [7].

In this problem, the boundary conditions (i.e. isotropic confinement of the sample) do not allow the shear strength of the material to be reached and the development of the localised gas pathway is therefore not straightforward. It is proposed to consider the presence of a preexisting fracture through the sample to better represent the development of these features. Moreover the experimental data acquisition of gas outflow under both increasing and 
decreasing gas pressures provides a framework to test the model under various hydraulic boundary conditions, which is rarely available.

This paper focuses mainly on the discussion of the numerical results and presents an indepth analysis on the influence of the different components of the embedded fracture model. The different couplings are added progressively to the model in order to determine the significance of each in the reproduction of the gas breakthrough phenomena. This study highlights therefore the main effects that need to be taken into consideration for gas migration processes. The paper proposes additional insight on the model performance in simulating the gas flow processes in initially saturated claystone and allows modelling strategies to be drawn when describing practical problems of gas flow.

\section{Long term laboratory gas injection test}

To investigate the gas migration mechanisms in clay-rich media, the British Geological Survey have performed a number of long-term laboratory-scale tests on samples of CallovoOxfordian claystone (COx), a candidate host rock for the disposal of radioactive waste in France. Test specimens were manufactured by a combination of dry core-drilling (with gas flushing and vacuum removal of fines), diamond slicing and surface grinding, or produced by machine lathing, a more sympathetic process which is less likely to induce damage within the material as a result of sample preparation. In this experimental system, the sample is subjected to an isotropic confining stress with injection and backpressures controlled through high precision syringe pumps. The experimental set up uses a novel feature, i.e. the use of porous annular guard-ring filters around the inflow and outflow filters (Figure 1). These allow the estimation of hydraulic anisotropy from a single test, as well as the monitoring of the pore pressure evolution in both guard-ring filters [25]. Testing is performed in an air-conditioned laboratory at a nominal temperature of $20^{\circ} \mathrm{C}$.

Prior to gas injection, each sample is first restressed with an isotropic confining pressure and a water pressure of 12.5 MPa and 4.5 MPa respectively. Confining pressure was applied in a series of steps in order to observe the consolidation response of the material. Once complete hydraulic testing was performed while the confining and backpressure were maintained constant. The water pressure at the injection filter was raised to $7.5 \mathrm{MPa}$ and held constant until steady state conditions were observed. Once complete, the injection pressure was reduced back to $4.5 \mathrm{MPa}$ ready for gas testing. The baseline hydraulic properties 
(intrinsic permeability and hydraulic anisotropy) may be determined during the hydraulic tests.

During the gas injection phase, helium injection occurs through the base of the sample by slowly increasing gas pressure in a series of steps (from 6.5 to $12 \mathrm{MPa}$ ) over a 600 days period and then by decreasing progressively the gas pressure (from 12 to $7 \mathrm{MPa}$ ). Finally, the injection pump was switched off and the gas pressure allowed decaying to define the apparent capillary threshold pressure. A backpressure of $4.5 \mathrm{MPa}$ is continuously imposed at the top of the sample through the outflow central filter and an isotropic confining stress of $12.5 \mathrm{MPa}$ is maintained during the entire experiment. All the experimental boundary conditions are summarized in Tables 1 and 2 .

The argillite specimen has an initial diameter of $54.4 \mathrm{~mm}$ and a height of $53.9 \mathrm{~mm}$. The orientation of the sample is perpendicular to the bedding. More details about the mineralogical composition of the COx can be found in [24].

A complete analysis of the experimental results can be found in [7]. However, presented here are simply the main experimental observations required for modelling the data measured at the injection and backpressure filters (Figure 2). A small emergent flux was observed during the early stages of testing, which was found to vary with the magnitude of the injection gas pressure. A possible interpretation is that this flux may be aqueous in nature relating to slug flow caused by residual water displaced from the injection filter. This was further supported by post-test measurements which showed no discernible desaturation (from an accurate measurement of the volume, using a digital micrometer, and the weight).

Major gas breakthrough occurred after 170 days of testing (Figure 2(a)), signified by a spontaneous increase in outflow, symptomatic of the development of conductive gas pathways. A quasi steady state was then reached (Figure 2(b)) around day 220. Some variation in gas discharge rates were then detected during additional increments in gas pressure, which can be interpreted as evidence of dynamic flow behaviour and timedependant propagation of gas pathway across the sample. However, such observations are difficult to explain with standard porous medium flow approaches.

Finally as injection gas pressure is reduced, the asymptote of flux for each subsequent steady state is significantly higher than that observed during the initial increasing injection pressure. Hysteresis behaviour on drainage and imbibition paths is common in two-phase flow processes [25] and can result from plastic strains around dilatant pathways, fabric alteration or incomplete resaturation. 


\section{Hydro-mechanical model}

Hereafter is presented a general framework for the modelling of unsaturated porous media in isothermal conditions. Only are presented the main balance and constitutive equations (see [26] for more details). First are recalled the fluid mass balance equations (balance of momentum equation is also taken into account). The constitutive equations are then defined. The emphasis is put on the hydro-mechanical coupling proposed to reproduce the development of preferential gas pathways in claystone.

Following the ideas of Panday and Corapcioglu [27] and Olivella et al. [28], the fluid mass balance equations are written for each chemical species (i.e. water and helium). In this way the terms related to the phase transfer cancel. The water and helium mass balance equations read:

$$
\begin{array}{r}
\underbrace{\operatorname{div}\left(\underline{f}_{w}\right)+\frac{\partial}{\partial t}\left(\rho_{w} \phi S_{r, w}\right)_{w}}_{\text {Liquid water }}+\underbrace{\operatorname{div}\left(\underline{f}_{v}\right)+\frac{\partial}{\partial t}\left(\rho_{v} \phi\left(1-S_{r, w}\right)\right)}_{\text {Water vapour }}-Q_{w}=0 \\
\underbrace{\operatorname{div}\left(\underline{f}_{H e}\right)+\frac{\partial}{\partial t}\left(\rho_{H e} \phi\left(1-S_{r, w}\right)\right)}_{\text {Dry helium in gaseous phase }}+\underbrace{\operatorname{div}\left(\underline{f}_{H e-d}\right)+\frac{\partial}{\partial t}\left(\rho_{H e-d} \phi S_{r, w}\right)}_{\text {Dissolved helium in water }}-Q_{H e}=0
\end{array}
$$

where $\rho_{w}, \rho_{v}, \rho_{H e}$ and $\rho_{H e-d}$ are the densities respectively of liquid water, water vapour, helium and dissolved helium; $\underline{f}_{l}, \underline{f}_{v}, \underline{f}_{H e}$ and $\underline{f}_{H e}$ are the total mass flow respectively for liquid water, water vapour, helium and dissolved helium; $Q_{w}$ and $Q_{H e}$ are the sink terms of water and helium.

\section{Two-phase flow model}

The different mass flows in partially saturated porous media are reproduced by a twophase flow model. This model is comprised of a liquid phase, composed of liquid water and dissolved helium and a gaseous phase, which is an ideal mixture of helium and water vapour. It takes into account the advection of each phase using the Darcy's law, including the permeability anisotropy associated with the bedding planes of the rock, as well as the diffusion of the components within each phase (Fick's law) (see [26] for more details). 


\section{Stress-strain behaviour}

While a constant isotropic confining stress is imposed to the sample during the experiment the stress state remains inside any yield criterion, which does not therefore play any role in the development of strains. Hence the mechanical model considered for COx argillite is a linear elastic model, written in terms of net stress.

\section{Embedded fracture model}

To reproduce the development of preferential gas pathways in COx, we consider that gas migration is associated with the development of preferential paths along existing or pressure-dependent discontinuities. The fracture opening can be induced by hydro-mechanical loadings, as an increase of fluid pressure or an effective unloading of the sample (i.e. a reduction of net stress). The basic idea of the model deals with the appropriate representation of these embedded fractures in a continuous finite element. A hydro-mechanical coupling between the pathways aperture, permeability and air entry pressure is proposed on the basis of the embedded fracture model [21]. The fracture aperture $b$ is introduced respectively in the cubic law and in the Laplace equation in order to express its influence on the permeability and the air-entry pressure. Permeability along the fracture $K_{i j}$ increases with the opening of these features (assuming laminar flow inside the fracture), whilst the gas entry pressure $P_{r}$ decreases simultaneously. As proposed by Olivella \& Alonso [21], it is possible to express the change in air-entry pressure according to the change in permeability. Both equations depend therefore on the same parameter $\lambda$, reflecting the density of fractures and the rugosity and initially introduced to take into account the discrepancy between the fluid flows predicted by the cubic law and observed in rough fractures. Finally, in the finite element code, this aperture $\mathrm{b}$ is linked with the strains in the discontinuities:

$$
\begin{gathered}
K_{i j}=K_{i j, \text { matrix }}+K_{0}\left(1+\lambda\left(\left\langle\varepsilon_{n}-\varepsilon_{0}\right\rangle\right)\right)^{3}\left(\delta_{i j}-n_{j} n_{i}\right) \\
P_{r}=P_{r, 0} \frac{1}{1+\lambda\left(\left\langle\varepsilon_{n}-\varepsilon_{0}\right\rangle\right)}
\end{gathered}
$$


and with $\varepsilon_{n}$ the normal strain to the gas pathway, $\varepsilon_{0}$ the threshold strain required to initiate fracture opening, $K_{i j \text {,matrix }}$ the permeability of the undisturbed claystone, $K_{0}$ and $P_{r, 0}$ respectively the initial permeability and gas entry pressure along the fracture and $\underline{n}$ the normal to the fracture (considered parallel to the maximum principal strain).

In addition to the initial model [21] it is proposed to include both an "opening onset" threshold strain $\varepsilon_{0}$ and a "maximal opening" threshold strain $\varepsilon_{1}$ for the fracture, such that we define:

$$
\left\langle\varepsilon_{n}-\varepsilon_{0}\right\rangle= \begin{cases}0 & \text { if } \varepsilon_{n}<\varepsilon_{0} \\ \varepsilon_{n}-\varepsilon_{0} & \text { if } \varepsilon_{0} \leq \varepsilon_{n} \leq \varepsilon_{1} \\ \varepsilon_{1}-\varepsilon_{0} & \text { if } \varepsilon_{n}>\varepsilon_{1}\end{cases}
$$

In this paper, this hydro-mechanical coupling is used to reproduce the development of the gas pathways within the claystone, as observed in the laboratory experiment. The proposal is to initiate gas flow through preferential paths by assuming initial fractures in the sample. Only the elements defining the initial discontinuity are activated whenever aperture changes are calculated. Given isotropic confinement pressure remains constant in the experiment, the preexisting discontinuity can thus only be opened due to an increase of fluid pressure.

\section{Boundary value problem}

A 2D axisymmetric simulation of the problem has been performed using the finite element code Lagamine [29], assuming isothermal conditions. Only the gas injection stages are considered, under both increasing and decreasing gas pressures. The sample, inflow and outflow filters ( $\mathrm{I}$ and $\mathrm{O}$ ) as well as the guard-ring filters (IG and $\mathrm{OG}$ ) are all taken into account within the model (Figures 1 and 3). The initial water pressure in the system is equal to 4.5 $\mathrm{MPa}$ and the initial gas pressure is equal to the atmospheric pressure.

Injection gas pressure is imposed at the inflow filter (I), whilst the backpressure (4.5 MPa) is maintained constant at the outflow filter $(\mathrm{O})$. An isotropic confinement pressure of 12.5 $\mathrm{MPa}$ is applied to each boundary, except at the bottom where the displacements are prescribed to zero. 
As explained previously a pre-existing fracture is included within the sample in order to allow the development of the gas pathways. This pre-existing fracture is allowed to open as gas pressure in the sample increases. While the geometry of the fracture is somewhat arbitrary (Figure 4), it is qualitatively based on the experimental data which shows sudden increases in gas pressure first at the injection guard-ring filter (IG), then at the outflow guard-ring filter (OG) and finally at the outflow filter (O) [7].

The origin of such pressurized fractures remains unclear. Discontinuities developed during the coring of the sample or initial heterogeneity of the material in pore size distribution can be explanations. Emerging techniques such as nanoparticle injection into the gas stream, combined with a post-test scanning electron microscope analysis, can be used as a method to study gas transport pathways through clay or mudrock [30]. Such kinds of methods will provide additional insights into the processes governing gas flow to help guide future numerical modelling.

\section{Hydro-mechanical properties}

The mechanical and hydraulic properties used for COx argillite for the simulation are presented in Table 3. The properties have been adopted from the pre-test estimation of the porosity [7] and from the numerical modelling of the preliminary consolidation stage and hydraulic tests for the hydro-mechanical properties under saturated conditions [31]. The water permeability under saturated conditions and its anisotropy have been obtained from the modelling of the hydraulic tests thanks to the porous annular guard-ring filters. Even if this anisotropy ratio shows further its relevance in the model, it is worth noting that smaller anisotropy ratio have been measured on other samples of Cox [7]. Some uncertainties remain therefore on this issue.

The unsaturated characteristics for COx (e.g. retention curve and evolution of the water ang as permeabilities with the degree of saturation) are based on existing experimental data [26]. Van Genuchen retention curve and water relative permeability relationship are defined, while a cubic law is adopted to describe the relative gas permeability:

$$
S_{r, w}=\left(1+\left(\frac{S}{P_{r}}\right)^{n}\right)^{\frac{1}{n}-1}
$$




$$
\begin{gathered}
K_{w}=K_{w}^{s a t} \sqrt{S_{r, w}}\left(1-\left(1-S_{r, w}^{1 / m}\right)^{m}\right)^{2} \\
K_{g}=K_{g}^{d r y}\left(1-S_{r, w}\right)^{3}
\end{gathered}
$$

where $S_{r, w}$ is the degree of saturation, $s$ the suction $\left(=p_{g}-p_{w}\right.$ with $p_{g}$ and $p_{w}$ the gas and water pressures), $K_{w}^{s a t}$ and $K_{g}^{d r y}$ the water and gas permeabilities respectively in saturated and dry conditions and $n$ and $m$ two parameters.

The filters have been modelled by means of an equivalent porous material having a high stiffness, a high permeability and porosity equal to 1 . The adopted retention curve has a very low air entry pressure and a flat shape (i.e. very fast saturation/desaturation).

\section{Numerical results}

To contribute to a better understanding of the processes associated with the development of localised gas pathways in claystone, the different couplings are added progressively to the model in order to highlight the influence of each in the reproduction of the gas migration mechanisms.

\section{1. $\quad$ Response of a two-phase flow model}

In order to illustrate the important role of the embedded fracture within the proposed model, a standard two-phase flow model (with a hydro-mechanical component) is first presented. Figure 5(a) shows a comparison between measured and computed flows at the outflow filter. While it suggests that a standard two-phase flow model allows the reproduction of the small outflow noted during the first stages of testing, it is not sufficient to model the strong rise of the measured outflow during the breakthrough (day 200). However, the numerical data provides some explanations about the origin of the volumetric flux at the outlet filter, which was not initially possible from the experimental results. While the syringe 
pump of the backpressure system measures volumetric flow rates, it cannot determine the nature of the outflow (water or helium).

The analysis of the fluxes at the outlet filter suggests that the flux is mainly aqueous in nature at the beginning (Figure 5(b)). After 120 days, gas flows increase at the bottom of the sample, whilst water flows decrease with the progressive expulsion of the interstitial water. The flux becomes progressively gaseous at the outflow filter. An in-depth analysis of the fluxes along a vertical section between the inlet and outlet filters shows that the water initially expelled is explained by the fast increase in gas pressure in the reservoir. The inflow filter is desaturated and the pressures are transferred to the water through the claystone pores, which explained the water outflow (Figure 6(a)). The model suggests also that a first gaseous arrival is obtained after 10 days. It corresponds to the diffusion of dissolved helium along the sample (Figure 6(b)). The influence of dissolved gas seems to be reduced as far as the injection continues (Figures 6(c-d)) and is explained by the progressive desaturation of the claystone.

This first modelling shows how the small emergent flux noted during the early stages of testing can be reproduced with a two-phase flow model. Moreover the nature of the outflow during these stages is now known: only water at the beginning, then a mixture of water and helium with an increasing proportion of the gaseous flow when gas injection increases and finally only a gaseous component at the outlet filter.

While aspects of the test can be modelled using such approaches, the numerical results also highlight how the gas breakthrough is difficult to explain with standard porous medium flow models. In this paper, it is proposed that a strong hydro-mechanical coupling could adequately explain the formation and subsequent behaviour of gas conductive pathways within the claystone.

\subsection{Response of the embedded fracture model without criterion of maximal opening}

To explain the gas breakthrough observations, the hydro-mechanical framework is expanded by including an additional hydro-mechanical coupling, termed the embedded fracture model (equations 1 to 3). A pre-existing fracture is assumed (Figure 4), which is allowed to open as fluid pressure rises. The geometry of the fracture is qualitatively based on the experimental data which shows sudden increases in gas pressure first at the injection guard-ring filter (IG), then at the outflow guard-ring filter (OG) and finally at the outflow filter $(\mathrm{O})$. In this section, the experimental data are compared with the numerical results 
obtained using this approach, but without the inclusion of a maximal opening criteria (as proposed initially [21]).

Hydro-mechanical modelling of the test is performed with the properties defined in Tables 3 and 4 . The $\lambda$ parameter reflecting the density of fractures and the rugosity is here considered as an adjustment parameter to better fit the experimental data. The pre-existing fracture is first closed due to the high confining stress, which it leads to assign the same permeability as that for the surrounding claystone at the beginning of the gas injection test. This assumption seems reasonable as it is generally considered that self-sealing processes in a wide variety of argillaceous rocks (plastic clays to indurated clays) leads to the recovery of the permeability at the laboratory scale [32-34]. In this case, the self-sealing is likely to be enhanced by both the application of the confining stress and the inflow of water during the hydraulic tests. However, few experiments have investigated the influence of the self-sealing on the gas entry pressure and some degree of uncertainty remains. However, experimental evidence seems to show the low influence of resaturation on the gas entry pressure of argillaceous samples initially fractured, so long as the confining stress remains at a moderate level $<12 \mathrm{MPa}[35]$. To reflect this behaviour, a lower gas entry pressure is assumed for the fracture (Tables 3 and 4).

The small emergent flux at the outlet filter is still reproduced by the two-phase flow component of the constitutive modelling (Figure 7(a)). The threshold strain is not reached during the first gas injection stages and the modification of the permeability and the gas entry pressure is therefore not activated. While gas injection pressure increases, the effective unloading of the sample induces strains higher than the threshold strain and the embedded fracture model becomes active. The spontaneous rise in gas outflow observed after 170 days can be now reproduced with the proposed hydro-mechanical couplings (Figure 7(a)). On the other hand, the same modelling has been performed using only the evolution of the permeability with the strains while maintaining a constant gas entry pressure. However, Figure 7(a) shows that such a coupling is not sufficient to reproduce the spontaneous increase in outflow. To better represent this behaviour within our model, it is therefore required to impose that both the permeability and the gas entry pressure evolve as a function of fracture aperture.

Despite the fairly good reproduction of the data during the first four injection steps, longterm predictions strongly overestimate the gas outflow during the next injection stages (Figure 7(b)). This can be explained by the continuous increase in permeability and subsequent 
decrease in gas entry pressure observed for each injection stage, which ultimately generates a pathway with excessive conductivity.

\subsection{Response of the embedded fracture model with criterion of maximal opening}

To avoid the overestimation of the gas outflow for long term predictions, the initial embedded fracture model [21] is modified. While gas migration is still associated with the development of preferential paths along pressure-dependent discontinuities, a maximal opening criteria for the embedded fracture is now included through a "maximal opening" threshold strain $\varepsilon_{1}$ (equation 3). This approach limits the increase of the permeability and the decrease of the gas entry pressure, as it allows the progressive closure of the fracture to occur as gas pressure reduces. With the set of parameters proposed in Table 5, it is now possible to obtain a good agreement with long-term experimental observations (Figure 8(a)). Moreover the predicted flow rates illustrate that the gas outflow becomes quickly the only component of the emergent flux at the outlet filter. This is why no distinction is made hereafter between the total and gas outflows.

Using this model the injection flow rate is well reproduced for the latter stages of the test, but is underestimated for low gas injection pressures (Figure 8(b)). This highlights one of the main limitations of the model. The unstable dynamic behaviour of the pathways is difficult to reproduce. Our model provides an instantaneous increase of the permeability, while the experimental data suggests a more progressive development of the preferential pathways actually occurs. As such, the onset of the breakthrough is not particularly well represented for the same reasons. Nevertheless the general gas behaviour is well predicted with the embedded fracture model.

An in-depth analysis of the results illustrates the evolution of the permeability and the gas entry pressure along the fracture. It shows that the increase in permeability and decrease in gas entry pressure take place close to the injection filter as soon as the gas injection begins (Figure 9). It then progresses towards the end of the sample. The threshold strain required to initiate a fracture across the entire length of the sample does not occur until the third injection test stage. The opening of the conductive pathway stops at the beginning of the fourth injection step (day 240), as the permeability increases corresponds to about two orders of magnitude (Figure 9(a)). The ratio between the initial and the final gas entry pressure is about 5 (Figure 9(b)), which influences the retention behaviour of the claystone (Figure 9(c)). 
A salient finding is that the gas flows in the COx claystone sample can be simply characterized by two conductivities. The initial conductivity corresponds to that of intact claystone, which then allows prediction of the small emergent flow observed at the beginning of the gas injection test. When the gas breakthrough occurs, the conductivity increases and quickly reached its maximum value. The model suggests that the last gas injection steps can be explained by a constant "adapted" conductivity of the medium in a standard two-phase flow framework.

\subsection{Influence of the gas pathway geometry}

While the hydro-mechanical model leads to convincing numerical results, they remain arbitrary, dependent on the definition of the initial fracture geometry. Although its definition is based on experimental evidence (Figure 4), the exact geometry, thickness and number of pathways is unknown. This section is therefore devoted to the analysis of the gas pathway geometry on the numerical predictions. With this in mind, a simple case is considered whereby a vertical gas pathway connects both the inlet and outlet filters (Figure 10).

Figure 11(a) shows that it is still possible to reproduce the experimental outflow with the simplified fracture geometry and using a set of parameters close to the first one (Table 6). The subsequent numerical results are in good agreement with the experimental inflow rate (Figure 11(b)).

These results also demonstrate the ability of the model to reproduce the closure of the fracture during decreasing gas injection stages. As the stress state remains in the elastic domain, it leads to a total recovery of the system (in terms of permeability and the gas entry pressure) when gas injection ceases. However, to achieve a good fit to the data, a small reduction in fracture density $\lambda$ is required. This makes it possible to resolve the highly coupled boundary conditions, central to which is an evolution in permeability.

An analysis of the results obtained with these two fracture geometries should lead to the conclusion that the average conductivity of the sample evolve similarly. However, this assumption is difficult to verify, because the injection plate covers only a small section of the sample face. Owing to these particular gas injection conditions, all the elements of the mesh do not contribute with the same weight to the average sample conductivity. Nevertheless this result shows the performance of the model, whatever the geometry of the pre-existing fracture, to reproduce the development of localised pathways in initially saturated samples subjected to gas injection. Even if it does not, at present, provide a predictive modelling tool, 
such an approach illustrates the importance of the hydro-mechanical couplings and pressuredependent discontinuities to describe localised gas migration in an initially saturated claystone.

\section{Conclusions}

The modelling of gas migration in clayey materials remains an issue, because gas injection experiments highlight discontinuous phenomena such as the development of dynamic and localized gas pathways through the sample. Such behavior is difficult to reproduce with classic visco-capillary (two-phase) flow models.

In this paper a 2D axisymetrical model of a long-term laboratory helium injection experiment is presented within the framework of a general hydro-mechanical model for geological media. The presence of a pre-existing embedded fracture is included within the model to reflect localisation of flow and provide a numerical framework for the hydromechanical coupling. The opening of the fracture is induced by the increase in fluid pressure. By considering the simultaneous evolution of the permeability and the retention curve along this fracture with the strains, the hydro-mechanical model yields a better description of the mechanisms governing gas flow within claystone. Simulations show a good agreement with the global behaviour of the observed gas outflows, for both increasing and decreasing gas pressure stages.

The discussion of the numerical results offers an insight into some of the open questions stemming from analysis of the experimental data:

- The numerical predictions provide information on the potential nature of the small emergent flux at the outlet filter at the beginning of the test. This small emergent flux, which is first aqueous in nature, may relate to slug flow caused by residual water displaced from the injection filter to the sample. The first gaseous arrival is obtained after 10 days and is due to the diffusion of dissolved helium. Thereafter, the gaseous component of the outflow gradually increases, before becoming the only component of flow from the spontaneous increase in outflow observed on day 200.

- A two-phase approach allows the reproduction of the experimental data at the beginning of the gas injection. As gas pressure increases, complex behaviour for gas flow is observed, associated with the opening of pressure-dependent gas pathways inside the matrix. The model suggests that the average transport properties of the sample are modified by the pressure rise only during a short period of time. After the breakthrough the new and adapted transport 
properties of claystone can be viewed again as invariants with the state of deformation of the rock. Using the new permeability and retention curve, a standard two-phase flow model is then sufficient to predict the gas outflow measurements for the highest injection pressures.

Moreover the numerical results allow some conclusions on modelling strategies to be drawn and their use in practical problems:

- The necessity to take into consideration not only the evolution of the permeability with the opening of the fracture, but also the modification of the gas entry pressure has been highlighted.

- The introduction of a criterion defining the maximum opening of the fracture is necessary to reproduce the long-term experimental observations. A two order of magnitude increase of permeability provides a good agreement with the experimental outflow.

- In the model the opening of the pathway is controlled by the threshold strain. The model response shows that the opening threshold strain is reached when a capillary pressure of 2 $\mathrm{MPa}$ is imposed at the injection filter. As gas migration progresses and gas pressure increases, the opening of the pathway grows and reaches the other face when a capillary pressure of 3 $\mathrm{MPa}$ is imposed at the injection filter. This range of suction corresponds to the range of gas entry pressures measured on COx samples [36].

Some improvements could be made to the hydro-mechanical model. 3D modelling of the experiment would allow the inclusion of a non-symmetrical geometry along the sample axis, which is certainly closer to the reality. Moreover the highly dynamic fluid flow responses can be improved by introducing time-dependent processes in the model (fabric alteration, self-sealing, creep behaviour, etc.) or the presence of several pre-existing fractures in the model (characterised by different air entry pressures or threshold strains), which would lead to successive opening of pathways during the gas injection. Nevertheless the current model highlights the importance of hydro-mechanical coupling and pressure-dependent features, and their relevance to explain gas flow through initially saturated claystone.

\section{Acknowledgements}

The research leading to these results has received funding from the European Atomic Energy Community's Seventh Framework Programme (FP7/2007-2011) under Grant Agreement no. 230357, the FORGE project. 


\section{References}

[1] Ortiz L., Volckaert G., Mallants D. Gas generation and migration in clay barriers. Eng. Geol. 2002;64:287-296.

[2] Marschall P., Horseman S.T., Gimmi T. Characterisation of Gas Transport Properties of the Opalinus Clay, a Potential Host Rock Formation for Radioactive Waste Disposal. Oil Gas Sci. Technol. 2005;60:121-139.

[3] Volckaert G., Ortiz L., de Cannière P., Put M., Horseman S.T., Harrington J.F., Fioravante V., Impey M. MEGAS : Modelling and Experiments on Gas Migration in Repository Host Rocks - Phase 1. Final Report. European Commission Report EUR $16235 \mathrm{EN} ; 1995$.

[4] Horseman S.T., Harrington J.F., Sellin P. Gas migration in clay barriers. Eng. Geol. 1999;54:139-149.

[5] Harrington J.F., Horseman S.T. Gas migration in KBS-3 buffer bentonite. Sensitivity of test parameters to experimental boundary conditions. Technical Report SKB - TR-03$02 ; 2003$.

[6] Arnedo D., Alonso E.E., Olivella S., Romero E. Gas injection tests on sand/bentonite mixtures in the laboratory. Experimental results and numerical modelling. Phys. Chem. Earth. 2008;33:237-247.

[7] Harrington J.F., de La Vaissière R., Noy D.J., Cuss R.J., Talandier J. Gas flow in CallovoOxfordian claystone $(\mathrm{COx})$ : results from laboratory and field-scale measurements. Mineral. Mag. 2012;76(8):3303-3318.

[8] Gallé C. Gas breakthrough pressure in compacted Fo-Ca clay and interfacial gas overpressure in waste disposal context. Appl. Clay Sci. 2000;17(1-2):85-97.

[9] Graham J., Halayko K.G., Hume H., Kirkham T., Gray M., Oscarson D. A capillarityadvective model for gas brak-through in clays. Eng. Geol. 2002;64:273-286.

[10] Angeli M., Soldal M., Skurtveit E., Aker E. Experimental percolation of supercritical $\mathrm{CO}_{2}$ through a caprock. Energy Procedia. 2009;1:3351-3358.

[11] Cuss R.J., Harrington J.F., Noy D.J., Wikman A., Sellin P. Large scale gas injection test (Lasgit): Results from two gas injection tests. Phys. Chem. Earth. 2011;36:1729-1742.

[12] de La Vaissière R., Talandier J. Gas entry pressure in Callovo-Oxfordian claystone : insitu experiment PGZ1. In: Proceedings of the Transfert 2012 conference. Lille, 2012. p. 360-368. 
[13] de La Vaissière R., Gerard P., Radu J.-P., Charlier R., Collin F., Granet S., Talandier J., Piedevache M., Helmlinger B. Gas injection test in the Callovo-Oxfordian claystone: data analysis and numerical modeling. In: Clays in Natural and Engineered Barriers for Radioactive Waste Confinement, Norris S., Bruno J., Cathelineau M., Delage P., Fairhurst C., Gaucher E. C., Höhn E. H., Kalinichev A., Lalieux P., Sellin P. editors. Geological Society, London, Special Publications, 2013; 400: http://dx.doi.org/10.1144/SP400.10

[14] Gerard P., Charlier R., Barnichon J.-D., Su K., Shao J.-F., Duveau G., Giot R., Chavant C., Collin F. Numerical modelling of coupled mechanics and gas transfer around radioactive waste in long-term storage. J. Theor. Appl. Mech. 2008;38(1-2):25-44.

[15] Duveau G., M’Jahad S., Davy C.A., Skoczylas F., Shao J.-F., Talandier J., Granet S. Gas entry through water-saturated argillite: experimental and numerical approaches. In: Proceedings of 45th US Rock Mechanics/Geomechanics Symposium. San Francisco; 2011. p. 53-60.

[16] Grindrod P., Impey M.D., Saddique S.N., Takase H. Saturation and gas migration within clay buffers. In: Proceedings of Conference on High Level Radioactive Waste Management. Las Vegas; 1994. p. 2069-2075.

[17] Xu K., Daian J.-F., Quenard D. Multiscale Structures to Describe Porous Media. Part I : Theoretical Background and Invasion by Fluids. Transport Porous Med. 1997;26(1):5173.

[18] Xu K., Daian J.-F., Quenard D. Multiscale Structures to Describe Porous Media. Part II : Transport properties and application to test materials. Transport Porous Med. 1997;26(3):319-338.

[19] Zimmerman R.W., Bodvarsson G.S. Effective transmissivity of a two-dimensional fracture network. Int. J. Rock Mech. Min. Sci. 1996;33:433-438.

[20] Delahaye C.H., Alonso E.E. Soil heterogeneity and preferential paths for gas migration. Eng. Geol. 2002;64:251-271.

[21] Olivella S., Alonso E.E. Gas flow through clay barriers. Géotechnique. 2008;58:157-168.

[22] Arnedo D., Alonso E.E., Olivella S. Gas flow in anisotropic claystone: modelling triaxial experiments. Int. J. Numer. Anal. Met. 2013;37:2239-2256.

[23] Delay J., Vinsot A., Krieguer J.M., Rebours H., Armand G. Making of the underground scientific experimental programme at the Meuse/Haute-Marne underground research laboratory, North Eastern France. Phys. Chem. Earth. 2007;32:2-18. 
[24] Wileveau Y., Bernier F. Similarities in the hydromechanical response of CallovoOxfordian clay and Boom Clay during gallery excavation. Phys. Chem. Earth. 2008;33:S343-S349.

[25] Harrington J.F., Noy D.J., Horseman S.T., Birchall J., Chadwick R. Laboratory study of gas and water flow in the Nordland Shale, Sleipner, North Sea. In: Carbon dioxide sequestration in geological media-State of the science. Grobe M., Pashin J.C., Dodge R.L., editors. 2009. p. 521- 543.

[26] Charlier R., Collin F., Pardoen B., Talandier J., Radu J.-P., Gerard P. An unsaturated hydro-mechanical modelling of two in-situ experiments in Callovo-Oxfordian argillite. Eng Geol. 2013;165:46-63.

[27] Panday S., Corapcioglu M.Y. Reservoir transport equations by compositional approach. Transport Porous Med. 1989;4:369-393.

[28] Olivella S., Carrera J., Gens A., Alonso E.E. Non isothermal multiphase flow of brine and gas through saline media. Transport Porous Med. 1994;15:271-293.

[29] Collin F., Li X.L., Radu J.-P., Charlier R. Thermo-hydro-mechanical coupling in clay barriers. Eng. Geol. 2002;64:179-193.

[30] Harrington J.F., Milodowski A.E., Graham C.C., Rushton J.C., Cuss R. Evidence for gasinduced pathways in clay using a nanoparticle injection technique. Mineral. Mag. 2012;76(8) :3327-3336.

[31] Gerard P. Impact des transferts de gaz sur le comportement poro-mécanique des matériaux argileux. PhD. Thesis. Université de Liège, Belgium ; 2011.

[32] Gutierrez M., Oino L.E., Nygard R. Stress-dependent permeability of a de-mineralised fracture in shale. Mar. Petrol. Geol. 2000;17:895-907.

[33] Davy C.A., Skoczylas F., Barnichon J.-D., Lebon P. Permeability of macro-cracked argillite under confinement: Gas and water testing. Phys. Chem. Earth. 2007;32:667680 .

[34] Zhang C.L., Rothfuchs T. Damage and sealing of clay rocks detected by measurements of gas permeability. Phys. Chem. Earth.. 2008 ;33 :S363-S373.

[35] M'Jahad S. Impact de la fissuration sur les propriétés de rétention d'eau et de transport des géomatériaux. Application au stockage profond des déchets radioactifs. $\mathrm{PhD}$ Thesis. Ecole centrale de Lille, France ; 2012.

[36] Andra. Rapport de mi-parcours pour le Groupement de Laboratoires « Transfert de gaz ». Rapport interne C.RP.ASCM09.0002; 2009. 


\begin{tabular}{c|c|c|c|c|c}
\hline & \multicolumn{3}{|c|}{ CONSOLIDATION } & \multicolumn{2}{c}{ HYDRO TESTS } \\
\hline Time (day) & $-41 \rightarrow-26$ & $-26 \rightarrow-21$ & $-21 \rightarrow-13$ & $-13 \rightarrow-5.4$ & $-5.4 \rightarrow 0$ \\
\hline $\begin{array}{c}\text { Isotropic confining } \\
\text { pressure (MPa) }\end{array}$ & 9.5 & 11 & 12.5 & 12.5 & 12.5 \\
\hline $\begin{array}{c}\text { Injection water } \\
\text { pressure (MPa) }\end{array}$ & 4.5 & 4.5 & 4.5 & 7.5 & 4.5 \\
\hline Backpressure (MPa) & 4.5 & 4.5 & 4.5 & 4.5 & 4.5 \\
\hline
\end{tabular}

Table 1 : Experimental boundary conditions of the consolidation phase and the hydraulic tests: confining pressure, backpressure and gas injection pressure

\begin{tabular}{|c|c|c|c|c|c|c|c|c|c|c|c|}
\hline & \multicolumn{11}{|c|}{ HELIUM INJECTION TESTS } \\
\hline Time (day) & $\begin{array}{c}0 \\
\rightarrow \\
32.8\end{array}$ & $\begin{array}{c}32.8 \\
\rightarrow \\
119\end{array}$ & $\begin{array}{c}119 \\
\overrightarrow{240}\end{array}$ & $\begin{array}{c}240 \\
\rightarrow \\
335\end{array}$ & $\begin{array}{c}335 \\
\rightarrow \\
426\end{array}$ & $\begin{array}{c}426 \\
\rightarrow \\
499\end{array}$ & $\begin{array}{c}499 \\
\rightarrow \\
596\end{array}$ & $\begin{array}{c}596 \\
\rightarrow \\
642\end{array}$ & $\begin{array}{c}642 \\
\rightarrow \\
681\end{array}$ & $\begin{array}{c}681 \\
\rightarrow \\
716\end{array}$ & $\begin{array}{c}716 \\
\rightarrow \\
898\end{array}$ \\
\hline $\begin{array}{l}\text { Isotropic confining } \\
\text { pressure }(\mathrm{MPa})\end{array}$ & 12.5 & 12.5 & 12.5 & 12.5 & 12.5 & 12.5 & 12.5 & 12.5 & 12.5 & 12.5 & 12.5 \\
\hline $\begin{array}{l}\text { Injection gas } \\
\text { pressure (MPa) }\end{array}$ & 6.5 & 7.0 & 7.5 & 8.0 & 8.5 & 9.0 & 10.5 & 12 & 9 & 7 & - \\
\hline $\begin{array}{l}\text { Backpressure } \\
(\mathrm{MPa})\end{array}$ & 4.5 & 4.5 & 4.5 & 4.5 & 4.5 & 4.5 & 4.5 & 4.5 & 4.5 & 4.5 & 4.5 \\
\hline
\end{tabular}

Table 2 : Experimental boundary conditions of the gas injection tests: confining pressure, backpressure and gas injection pressure

\begin{tabular}{ll|lc}
\hline Hydraulic parameter & Value & Mechanical parameter & Value \\
\hline $\begin{array}{l}\text { Horizontal water permeability in saturated } \\
\text { conditions, } K_{w, h}^{\text {sat }}\left(\mathrm{m}^{2}\right)\end{array}$ & $310^{-20}$ & Young's modulus, $E(\mathrm{MPa})$ & 3800 \\
Vertical water permeability in saturated conditions, $K_{w, v}^{s a t}$ & $1.710^{-21}$ & Poisson's ratio, $v(-)$ & 0.3 \\
$\left(\mathrm{~m}^{2}\right)$ & & & \\
Horizontal gas permeability in dry conditions, $K_{g, h}^{d r y}\left(\mathrm{~m}^{2}\right)$ & $310^{-19}$ & Cohesion, $c(\mathrm{MPa})$ & 3 \\
Vertical gas permeability in dry conditions, $K_{g, v}^{d r y}\left(\mathrm{~m}^{2}\right)$ & $1.710^{-20}$ & Friction angle, $\varphi\left(^{\circ}\right)$ & 20 \\
Initial porosity, $\phi_{i n i}(-)$ & 0.149 & & \\
Tortuosity, $\tau(-)$ & 0.30 & & \\
van Genuchten coefficient, $m(-)$ & 0.55 & & \\
van Genuchten coefficient, $n(-)$ & 1.49 & & \\
van Genuchten parameter, $P_{r}(\mathrm{MPa})$ & 15 & & \\
\hline
\end{tabular}

Table 3 : Hydro-mechanical properties of $\mathrm{COx}$ argillite 


\begin{tabular}{c|c}
\hline Parameter & Value \\
\hline Fracture density, $\lambda(-)$ & 5000 \\
Minimal Threshold strain, $\varepsilon_{0}(-)$ & -0.00064 \\
Initial gas entry pressure along the fracture, $P_{r, 0}(\mathrm{MPa})$ & 8 \\
\hline
\end{tabular}

Table 4 : Embedded fracture model properties with inclined fracture and without criterion of maximal opening

\begin{tabular}{c|c}
\hline Parameter & Value \\
\hline Fracture density, $\lambda(-)$ & 6000 \\
Minimal Threshold strain, $\varepsilon_{0}(-)$ & -0.00064 \\
Maximal Threshold strain, $\varepsilon_{1}(-)$ & -0.00049 \\
Initial gas entry pressure along the fracture, $P_{r, 0}(\mathrm{MPa})$ & 5 \\
\hline
\end{tabular}

Table 5 : Embedded fracture model properties with inclined fracture and a criterion of maximal opening

\begin{tabular}{c|c}
\hline Parameter & Value \\
\hline Fracture density, $\lambda(-)$ & 7000 \\
Minimal Threshold strain, $\varepsilon_{0}(-)$ & -0.00064 \\
Maximal Threshold strain, $\varepsilon_{l}(-)$ & -0.00049 \\
Initial gas entry pressure along the fracture, $P_{r, 0}(\mathrm{MPa})$ & 4 \\
\hline
\end{tabular}

Table 6 : Embedded fracture model properties with vertical fracture and a criterion of maximal opening

Figure 1: Scheme of the experimental device

Figure 2: (a) Outflow rate at the outflow filter and gas pressure at the injection filter - (b) Inflow rate at the injection filter and outflow rate at the outflow filter under SPT conditions during the gas injection test.

Figure 3: Geometry of the problem

Figure 4: Initial fracture in sample

Figure 5: Outflow: (a) comparison between experimental and numerical results with 2-phase flow model - (b) Distinction between water and gas outflows

Figure 6: Water, total gas and dissolved gas flows along a vertical cross-section between the inlet and outlet filters obtained with 2-phase flow model after (a) 10 days, (b) 90 days, (c) 130 days and (d) 300 days

Figure 7: Outflow: comparison between experimental and numerical results with embedded fracture model (without considering a maximal opening) (a) Influence of permeability and gas entry pressure evolutions with strains - (b) Long-term predictions when both permeability and gas entry pressure evolutions are activated

Figure 8: (a) Outflow: comparison between experimental and numerical results- (b) Inflow: comparison between experimental and numerical results with embedded fracture model

Figure 9: Prediction of the embedded fracture model with criterion of maximal opening. (a) Permeability with time, (b) Gas entry pressure evolution with time and (c) Retention curve evolution during the gas injection stages at different elements along the conductive gas pathway - (d) Definition of the elements

Figure 10: Vertical fracture in sample

Figure 11: (a) Outflow: comparison between experimental and numerical results- (b) Inflow: comparison between experimental and numerical results with embedded fracture model and a vertical conductive pathway 